\title{
Smoking is associated with an improved short-term outcome in patients with rib fractures
}

\author{
Areg Grigorian ${ }^{1}$ (D ) Michael Lekawa ${ }^{1} \cdot$ Matthew Dolich ${ }^{1} \cdot$ Sebastian D. Schubl ${ }^{1}$ Andrew R. Doben ${ }^{2}$. \\ Catherine M. Kuza ${ }^{3} \cdot$ Cristobal Barrios $^{1} \cdot$ Jeffry Nahmias ${ }^{1}$
}

Received: 21 February 2019 / Accepted: 13 May 2019 / Published online: 18 May 2019

○) Springer-Verlag GmbH Germany, part of Springer Nature 2019

\begin{abstract}
Background Smokers with cardiovascular disease have been reported to have decreased mortality compared to non-smokers. Rib fractures are associated with significant underlying injuries such as lung contusions, lacerations, and/or pneumothoraces. We hypothesized that blunt trauma patients with rib fractures who are smokers have decreased ventilator days and risk of in-hospital mortality compared to non-smokers.

Study design The Trauma Quality Improvement Program (2010-2016) was queried for patients presenting with a blunt rib fracture. Patients that died within $24 \mathrm{~h}$ of admission were excluded. A multivariable logistic regression model was performed. Results From 282,986 patients with rib fractures, 57,619 (20.4\%) were smokers. Compared to non-smokers with rib fractures, smokers had a higher median injury severity score (17 vs. $16, p<0.001)$. Smokers had a higher rate of pneumonia (7.5\% vs. $6.6 \%, p<0.001)$, however, less ventilator days ( 5 vs. $6, p=0.04)$, and lower in-hospital mortality rate $(2.3 \%$ vs. $4.6 \%, p<0.001)$, compared to non-smokers. After controlling for covariates, smokers with rib fractures were associated with a decreased risk for in-hospital mortality compared to non-smokers with rib fractures (OR 0.64, 0.56-0.73, $p<0.001$ ). Conclusion Despite having more severe injuries and increased rates of pneumonia, smokers with rib fractures were associated with nearly a $40 \%$ decreased risk of in-hospital mortality and one less ventilator day compared to non-smokers. The long-term detrimental effects of smoking have been widely established. However, the biologic and pathophysiologic adaptations that smokers have may confer a survival benefit when recovering in the hospital from chest wall trauma. This study was limited by the database missing the number of pack-years smoked. Future prospective studies are needed to confirm this association and elucidate the physiologic mechanisms that may explain these findings.
\end{abstract}

Keywords Trauma $\cdot$ Smoking $\cdot$ Nicotine $\cdot$ Mortality $\cdot$ Pneumonia

This work was presented at the 90th Annual Meeting of the Pacific Coast Surgical Association, Tucson, Arizona, February 2019.

Areg Grigorian

agrigori@uci.edu

1 Division of Trauma, Burns and Surgical Critical Care, Department of Surgery, Irvine Medical Center, University of California, 333 The City Blvd West, Suite 1600, Orange, CA 92868-3298, USA

2 Department of Surgery, Baystate Medical Center Affiliate of Tufts University School of Medicine, Springfield, MA, USA

3 Department of Anesthesiology, University of Southern California, Los Angeles, CA, USA

\section{Introduction}

Smoking continues to be a top public health epidemic. Although there has been a significant decline in per capita consumption of smoking tobacco, almost 25 trillion cigarettes have been consumed since the 1960s [1]. More than $30 \%$ of avoidable lung cancers and cardiac deaths have been attributed to smoking [2, 3]. For each person in the United States that succumbs to the ill effects of smoking, there are two new, young replacement smokers [4]. The prevalence of smoking is greatest in those with lower education, lower socioeconomic status, mental illness, and racial minority groups [5]. However, smoking does not uniformly and consistently affect all patient groups the same. More than 30 years ago, Kelly et al. reported lower mortality rates in patients with acute myocardial infarction who smoke 
compared to non-smokers [6]. This phenomenon, termed the "smoker's paradox", has been demonstrated in multiple subsequent studies on cardiovascular disease [7-9]. The mechanism is postulated to be multifactorial and related to changes in oxygen delivery, endothelial function, enhanced thrombolysis and inflammation; however, no conclusive data have been reported $[10,11]$.

Patients with fatal cardiac disease often have inadequate delivery and utilization of oxygen and the pathophysiologic adaptations in smokers may provide a survival benefit in this regard [12, 13]. Similarly, trauma patients have significant morbidity and mortality resulting from hypoxemia secondary to hypovolemia, hypotension, and/or respiratory failure $[14,15]$. Thus, the trauma population, in which there is a high smoking prevalence ranging from 22 to $43 \%$, may similarly benefit from the smoker's paradox [16, 17]. Ferro et al. reported that trauma patients who smoke had no difference in the rate of sepsis, respiratory complications, multiorgan failure, or mortality compared to non-smokers [17]. In a subsequent study, using a large national database, Bell et al. demonstrated that trauma patients aged $\leq 65$ years who smoke have a significantly less risk of mortality and major complications compared to non-smokers [18]. Both these studies included patients with a wide spectrum of injuries. Trauma patients involved in mechanisms severe enough to cause a rib fracture often have underlying lung damage (contusion, laceration, and/or pneumothorax) with subsequent pulmonary morbidity $[19,20]$. The smoker's paradox has not been evaluated in patients with rib fractures. We hypothesized that blunt trauma patients with rib fractures who are smokers have decreased ventilator days and associated with a decreased risk of mortality compared to non-smokers. We also sought to confirm the smoker's paradox in a subset of patients with coronary artery disease (CAD).

\section{Methods}

This study was approved by the Institutional Review Board at the Universality of California, Irvine. A retrospective analysis of the Trauma Quality Improvement Program (TQIP) was performed between January 2010 and December 2016. All patients $\geq 18$ years of age with one or more rib fractures after blunt trauma were identified using the International Classification of Diseases version-9 (ICD-9) diagnosis codes: 807-807.19 and 807.4. Patients that were current smokers were compared to non-smokers. This is listed as one of the 30-required comorbidities to be reported in the TQIP and is defined as "A patient who reports smoking cigarettes every day or some days within the last 12 months. Exclude patients who smoke cigars or pipes or use smokeless tobacco (chewing tobacco or snuff)." The primary outcomes were ventilator days and in-hospital mortality. Secondary outcomes included total hospital length of stay (LOS), intensive care unit (ICU) LOS, blood products transfused, thoracotomy for hemorrhage control, and in-hospital complications including pneumonia, acute kidney injury (AKI), and acute respiratory distress syndrome (ARDS).

Demographic variables collected included age, comorbidities, gender, the lowest systolic blood pressure within $24 \mathrm{~h}$ of admission, injury severity score (ISS), incidence of associated lung or heart injuries, flail chest, and abbreviated injury scale (AIS) for the head, spine, thorax and abdomen. Lung or cardiac injuries were identified by the appropriate ICD-9 diagnosis codes. Lung injuries were defined as lung contusion, laceration, pneumothorax, and/or hemothorax. All variables were coded as present or absent. Frequency statistics were performed for all variables. A Student's $t$ test or Mann-Whitney $U$ test was used to compare continuous variables and Chi square was used to compare categorical variables for bivariate analysis. Categorical data were reported as percentages, and continuous data were reported as medians with interquartile range or as means with standard deviation.

The magnitude of the association between predictor variables and mortality was first measured using a univariable logistic regression model. Covariates were chosen based on a review of the literature and included hypotension (systolic blood pressure $\leq 90 \mathrm{mmHg}$ ) within $24 \mathrm{~h}$, age $\geq 65$ years, ISS $\geq 25$, massive blood product transfusion ( $\geq 6$ units of packed red blood cells within $4 \mathrm{~h}$ ), severe (grade $>3$ ) AIS for the head, spine, thorax, abdomen, in-hospital AKI, pneumonia, ARDS, thoracotomy, and history of diabetes, hypertension, chronic obstructive pulmonary disease (COPD), and cerebrovascular accident (CVA). Covariates with statistical significance $(p<0.20)$ were included in a hierarchical multivariable logistic regression model and the adjusted risk for mortality was reported with an odds ratio (OR) and 95\% confidence intervals (CI). The reference group used in our analysis included blunt trauma patients with one or more rib fractures who were non-smokers. We also performed a subset analysis on patients with coronary artery disease (CAD), defined by those with history of myocardial infarction or angina within the previous 30 days. All $p$ values were two sided, with a statistical significance level of $<0.05$. All analyses were performed with IBM SPSS Statistics for Windows (Version 24, IBM Corp., Armonk, NY, USA).

\section{Results}

\section{Demographics and primary outcomes}

From 282,986 patients with rib fractures, 57,619 (20.4\%) were smokers and 225,367 (79.6\%) were non-smokers. Compared to non-smokers, those who smoked were younger 
(median age, 48 versus 54 years, $p<0.001$ ), more often male (75.3\% versus $66.8 \%, p<0.001)$, and had a higher median ISS (17 versus $16, p<0.001)$. The smoker group had lower rates of diabetes $(9.2 \%$ versus $13.6 \%, p<0.001)$ and hypertension $(25.4 \%$ versus $33.6 \%, p<0.001)$, but a higher rate of COPD $(11.7 \%$ versus $6.6 \%, p<0.001)$, compared to nonsmokers. They also had a higher rate of associated lung injuries $(63.3 \%$ versus $59.9 \%, p<0.001)$, similar rate of flail chest $(p=0.94)$, cardiac injuries $(p=0.18)$, and a lower rate of multiple rib fractures ( $83.0 \%$ versus $83.4 \%, p=0.01$ ), compared to non-smokers (Table 1). Smokers had less ventilator days (median, 5 versus $6, p=0.009$ ) and a lower rate of in-hospital mortality $(2.2 \%$ versus $4.6 \%, p<0.001)$, compared to non-smokers. In a subset of patients with CAD $(n=3870)$, the in-hospital mortality rate was lower in smokers compared to non-smokers ( $5.0 \%$ versus $7.7 \%, p=0.018$ ) but both groups had similar ventilator days (median, 6 days, $p=0.60$ ).

\section{Risk of mortality in trauma patients with rib fracture}

In a univariable logistic regression model, smoking was associated with a lower in-hospital mortality risk (OR 0.48 , CI $0.45-0.51, p<0.001)$. The strongest variable associated with in-hospital mortality was AKI (OR 12.38, CI
11.60-13.21, $p<0.001)$, followed by severe AIS of the head (OR 7.26, CI 7.00-7.54, $p<0.001$ ). After adjusting for covariates in a multivariable logistic regression model, smoking was found to be independently associated with a lower risk for in-hospital mortality (OR 0.64, CI 0.56-0.73, $p<0.001)$. The strongest independent risk factor associated with an increased risk of in-hospital mortality remained AKI (OR 6.57, CI 5.60-7.70, $p<0.001$ ) followed by severe AIS of the head (OR 4.79, CI 4.33-5.29, $p<0.001$ ) (Table 2). In a subset of patients with CAD, the risk for in-hospital mortality was similar (OR 0.51 , CI $0.10-2.58, p=0.42$ ).

\section{Other clinical outcomes in trauma patients with rib fractures}

Compared to non-smoking blunt trauma patients with rib fractures, smokers had no difference in median total hospital LOS $(p=0.16)$ or ICU LOS $(p=0.37)$. The smoking cohort required less red blood cells (RBCs) transfused within $4 \mathrm{~h}$ of admission (mean, 3.4 versus 4.1 units, $p<0.001$ ), and had no difference in the rate of thoracotomies required for hemorrhage control $(p=0.09)$. Compared to non-smokers, those who smoked had a higher rate of pneumonia (7.5\% versus $6.6 \%, p<0.001)$ and a trend towards a lower rate of ARDS (2.2\% versus $2.3 \%, p=0.06)$.
Table 1 Demographics of adult blunt trauma patients with rib fractures

\begin{tabular}{lccc}
\hline Characteristic & Non-smoker $(n=225,367)$ & Smoker $(n=57,619)$ & $p$ value \\
\hline Age, year, median (IQR) & $54.0(39,68)$ & $48.0(34,57)$ & $<0.001$ \\
Male, $n(\%)$ & $150,576(66.8 \%)$ & $43,383(75.3 \%)$ & $<0.001$ \\
ISS, median (IQR) & $16.0(10,22)$ & $17.0(11,22)$ & $<0.001$ \\
Lowest SBP within 24 h, median (IQR) & $71.0(35,96)$ & $66.0(35,94)$ & $<0.001$ \\
Multiple $(\geq 2)$ rib fractures, $n(\%)$ & $188,041(83.4 \%)$ & $47,817(83.0 \%)$ & 0.01 \\
Flail chest, $n(\%)$ & $10,684(4.7 \%)$ & $2727(4.7 \%)$ & 0.94 \\
Comorbidities, $n(\%)$ & & & $<0.001$ \\
Congestive heart failure & $6555(2.9 \%)$ & $864(1.5 \%)$ & $<0.001$ \\
End-stage renal disease & $1920(0.9 \%)$ & $270(0.5 \%)$ & $<0.001$ \\
Diabetes & $30,682(13.6 \%)$ & $14,644(25.4 \%)$ & $<0.001$ \\
Hypertension & $75,660(33.6 \%)$ & $594(1.0 \%)$ & $<0.001$ \\
History of myocardial infarction & $2997(1.3 \%)$ & $50(0.1 \%)$ & 0.06 \\
Angina in previous 30 days & $315(0.1 \%)$ & $6716(11.7 \%)$ & $<0.001$ \\
COPD & $14,784(6.6 \%)$ & & $<.2 \%)$ \\
AIS (grade $>3), n(\%)$ & & $7018(12.2 \%)$ & $<0.001$ \\
Head & $34,842(15.5 \%)$ & $1099(1.9 \%)$ & 0.91 \\
Spine & $4315(1.9 \%)$ & $9303(16.1 \%)$ & $<0.001$ \\
Thorax & $38,216(17.0 \%)$ & $3006(5.2 \%)$ & $<0.001$ \\
Abdomen & $10,934(4.9 \%)$ & & $<0.001$ \\
Associated injuries, $n(\%)$ & $135,003(59.9 \%)$ & & 0.18 \\
Lung & $2575(1.1 \%)$ & & \\
Heart & & & \\
\hline
\end{tabular}

$I S S$ injury severity score, $I Q R$ interquartile range, $S B P$ systolic blood pressure, $C O P D$ chronic obstructive pulmonary disease, $A I S$ abbreviated injury scale 
Table 2 Multivariable logistic regression analysis for risk of mortality in adult blunt trauma patients with rib fractures

\begin{tabular}{lllr}
\hline Risk factor & OR & CI & $p$ value \\
\hline Smoker & 0.64 & $0.56-0.73$ & $<0.001$ \\
Hypotensive (SBP $<90 \mathrm{mmHg}$ ) within & 1.14 & $1.03-1.27$ & 0.011 \\
$\quad 24 \mathrm{~h}$ & & & \\
Age & 1.03 & $1.03-1.03$ & $<0.001$ \\
ISS $\geq 25$ & 3.45 & $3.07-3.88$ & $<0.001$ \\
PRBC transfusion $\geq 6$ units within $4 \mathrm{~h}$ & 2.24 & $1.98-2.53$ & $<0.001$ \\
Peripheral vascular disease & 1.78 & $0.97-3.26$ & 0.061 \\
End-stage renal disease & 2.78 & $1.84-4.20$ & $<0.001$ \\
Diabetes & 1.23 & $1.07-1.42$ & 0.004 \\
Hypertension & 0.74 & $0.66-0.83$ & $<0.001$ \\
Chronic obstructive pulmonary disease & 1.14 & $0.95-1.37$ & 0.160 \\
Severe AIS-abdomen (grade $>3$ ) & 0.88 & $0.77-1.00$ & 0.052 \\
Severe AIS-thorax (grade $>3$ ) & 1.33 & $1.20-1.47$ & $<0.001$ \\
Severe AIS-head (grade $>3$ ) & 4.79 & $4.33-5.29$ & $<0.001$ \\
Severe AIS-spine (grade $>3$ ) & 1.35 & $1.10-1.67$ & 0.004 \\
Acute kidney injury & 6.57 & $5.60-7.70$ & $<0.001$ \\
Pneumonia/VAP & 0.50 & $0.44-0.57$ & $<0.001$ \\
Acute respiratory distress syndrome & 2.24 & $1.88-2.67$ & $<0.001$ \\
Thoracotomy for hemorrhage control & 2.24 & $1.64-3.05$ & $<0.001$ \\
Male & 1.17 & $1.06-1.29$ & 0.002 \\
\hline
\end{tabular}

$S B P$ systolic blood pressure, ISS injury severity score, $P R B C$ packed red blood cell transfusion, VAP ventilator-associated pneumonia, AIS abbreviated injury scale

\section{Discussion}

This retrospective analysis using 7 years of data from the TQIP found the prevalence of smoking in blunt trauma patients with rib fractures to be $20 \%$. Interestingly, the smoker's paradox exists in this population as we found smokers with rib fractures to be associated with nearly a $40 \%$ decreased risk of in-hospital mortality compared to non-smokers, even after controlling for many well-known risk factors of mortality in trauma. Additionally, we found smokers to require one less ventilator day compared to non-smokers. The rate of pneumonia was higher in smokers (Table 3).

Smokers with cardiovascular disease have been demonstrated to have improved outcomes compared to non-smokers with cardiovascular disease in multiple reports [6-9]. Our study suggests the smoker's paradox may also exist in blunt trauma patients with rib fractures that are recovering in the hospital. Interestingly, in a subset analysis of these patients with $\mathrm{CAD}$, smoking did not confer a survival advantage after controlling for covariates. Rib fracture patients have three primary issues: (1) impaired gas exchange; (2) impaired breathing mechanics; and (3) hypoventilation/atelectasis due to pain [21]. There are several mechanisms to help explain our findings. Nicotine, a key component in most cigarettes sold in the United States, causes an increase in the activation of the sympathetic nervous system. The resultant catecholamine surge can increase the heart rate, blood pressure, and cardiac contractility - changes that may be beneficial in trauma patients presenting with hypovolemic shock and/ or respiratory failure [22]. Additionally, nicotine potentiates systemic vasoconstriction which may reduce blood loss and improve blood pressure and tissue oxygen delivery [23]. This theory is supported in our findings as smokers required fewer RBCs transfused within $4 \mathrm{~h}$ of admission despite having a lower median SBP. In pulmonary vasculature, nicotine acts as a vasodilator which can further improve oxygen
Table 3 Clinical outcomes in adult blunt trauma patients with rib fractures

\begin{tabular}{|c|c|c|c|}
\hline Outcome & Non-smoker $(n=225,367)$ & Smoker $(n=57,619)$ & $p$ value \\
\hline LOS, days, median (IQR) & $6.0(3,11)$ & $6.0(3,11)$ & 0.16 \\
\hline ICU, days, median (IQR) & $4.0(2,9)$ & $4.0(2,9)$ & 0.37 \\
\hline Ventilator, days, median (IQR) & $6.0(2,13)$ & $5.0(2,12)$ & 0.04 \\
\hline PRBC units transfused within $4 \mathrm{~h}$, mean (SD) & $4.1(5)$ & $3.4(4)$ & $<0.001$ \\
\hline Thoracotomy for hemorrhage control, $n(\%)$ & $286(0.1 \%)$ & $57(0.1 \%)$ & 0.09 \\
\hline \multicolumn{4}{|l|}{ Respiratory complications, $n(\%)$} \\
\hline Pneumonia/VAP & $14,825(6.6 \%)$ & $4312(7.5 \%)$ & $<0.001$ \\
\hline Acute respiratory distress syndrome & $5226(2.3 \%)$ & $1260(2.2 \%)$ & 0.06 \\
\hline \multicolumn{4}{|l|}{ Disposition, $n(\%)$} \\
\hline Home & $135,200(63.4 \%)$ & $40,972(74.1 \%)$ & $<0.001$ \\
\hline Acute care rehabilitation & $49,201(23.1 \%)$ & $9863(17.8 \%)$ & $<0.001$ \\
\hline Hospice & $1067(0.5 \%)$ & $137(0.2 \%)$ & $<0.001$ \\
\hline Skilled nursing facility & $27,640(13.0 \%)$ & $4308(7.8 \%)$ & $<0.001$ \\
\hline Mortality, $n(\%)$ & $10,414(4.6 \%)$ & $1304(2.3 \%)$ & $<0.001$ \\
\hline
\end{tabular}

$L O S$ length of stay, $I Q R$ interquartile range, $S D$ standard deviation, $I C U$ intensive care unit, $P R B C$ packed red blood cells, VAP ventilator-associated pneumonia 
utilization and alleviate the effects of pulmonary hypertension in critically ill patients [24]. Finally, although nicotine can cause chronic pain disorders, it may potentially decrease the severity of acute pain [25]. This physiologic adaptation would be beneficial in patients with parietal chest wall pain secondary to rib fractures allowing for deep breathing exercises and thereby improved oxygenation.

Smoking promotes both inflammatory and anti-inflammatory effects. Smoking one cigarette exposes the respiratory tract to $15,000-40,000 \mu \mathrm{g}$ of particulate matter [26]. This results in oxidant stress and activation of inflammatory mediators, cytokines, and neutrophils with heightened local immune function [27]. In a non-injured patient, these changes over time result in a wide range of adverse sequelae including the destruction of alveolar walls, emphysematous changes, and COPD [28, 29]. As expected, our study demonstrated a significantly larger incidence of COPD in the smoking group. In the injured patient, these adaptations may confer a survival benefit. In the lung, catecholamines can function as immunomodulators of innate and adaptive immune cells including alveolar macrophages which can result in anti-inflammatory immune responses [30-32]. Our study supports this as we found smokers to require one less ventilator day and had a trend towards a lower rate of ARDS compared to non-smokers, although the latter finding was not statistically significant. This conflicts with prior reports demonstrating increased incidence of ARDS in smokers presenting after blunt trauma [33, 34]. Resnick et al. reported that trauma patients who smoke admitted to a level I center to spend more days on a mechanical ventilator, compared to non-smokers [35]. However, these studies focused on all critically ill patients presenting after severe trauma suggesting that smoking may not confer a similar mortality benefit for all non-thoracic injuries. Future prospective studies are needed to determine risk of ARDS in a generalizable trauma population and evaluate for potential pathophysiologic differences between smokers and non-smokers with ARDS.

Several predictors of mortality have been identified for blunt trauma patients presenting with rib fractures. These include age $\geq 65$ years, hemopneumothorax, extremity fractures, and head injuries [36, 37]. However, these studies, as well as a large systematic review and meta-analysis, which reported the predictors of mortality in patients with chest wall trauma, did not address how smoking affects mortality [38]. Our study is the first to demonstrate smoking to be associated with a significantly lower rate of in-hospital mortality in blunt trauma patients with rib fractures. While the intricacies of this association will need to be examined in the laboratory, there is a possible explanation. The leading cause of death in trauma patients surviving the initial resuscitation is sepsis and multiorgan failure [39]. Although this involves a complex interplay of inflammatory mediators, receptors and cytokines, none have proved to be a useful clinical target for intervention [40, 41]. Nicotine is considered a strong agonist for the alpha-7 nicotinic acetylcholine receptor (a7NAR), and has been demonstrated to attenuate the febrile response to lipopolysaccharide promoting an anti-inflammatory effect [42]. In mouse models, nicotine activating a7NAR has been demonstrated to improve survival in mice induced with sepsis by cecal ligation and puncture [43]. In vivo, nicotine attenuates cytokine release from macrophages improving survival in experimental models of sepsis suggesting a potential target to be exploited in the treatment of inflammatory disorders [44]. While we would not advocate for patients to smoke to prevent worse outcomes after trauma, future basic science research is needed to evaluate which components of smoking contribute to the protective effect that smoking confers to patients with rib fractures. This may allow the development of medications that augment respiratory and/or inflammatory pathophysiology in a protective manner.

Our study involves a large national database with multiple participating trauma centers; therefore, a reporting bias and coding errors are undoubtedly present. Furthermore, as a database, there were pertinent missing variables including the severity of rib fractures, such as whether the fractures were displaced or not, baseline arterial blood gas results, as well as pulmonary functional status (i.e., baseline incentive spirometer or pulmonary function testing) and interventions for analgesia such as rib fixation or an epidural catheter. In addition, limitations of the database included the number of cigarettes smoked daily as well as how many pack-years of smoking were present. Also, although the nonsmoking group had not smoked within the past 12 months, the percentage of ex-smokers in this group is not available within the database. We were also not able to determine if nicotine was in the system at the time of trauma and if any changes were a result of acute nicotine withdrawal as use of nicotine-replacement therapy is also not available within the database. The cause of death is not available with the TQIP database and only information pertaining to the indexhospitalization is available. As such, we are unable to report 30-day outcomes.

\section{Conclusion}

Despite having more severe injuries and increased rates of pneumonia and hypotension, smokers with rib fractures were associated with nearly a $40 \%$ decreased risk of in-hospital mortality compared to non-smokers. Additionally, they required one less ventilator day and had no difference in total and ICU LOS. The long-term detrimental effects of smoking have been widely established. However, biologic and pathophysiologic adaptations that smokers develop may provide a survival benefit when recovering from rib fractures 
after blunt trauma. Future prospective studies are needed to confirm this association, as well as basic science studies to elucidate the physiologic mechanisms that may explain these findings and develop therapeutic interventions without the long-term deleterious effects of cigarette smoking.

\section{Compliance with ethical standards}

Conflict of interest Areg Grigorian, Michael Lekawa, Matthew Dolich, Sebastian D Schubl, Andrew R Doben, Cathertine M Kuza, Cristobal Barrios, and Jeffry Nahmias declare that they have no conflict of interest.

Ethical statement This research involved humans. However, since this retrospective study was performed using a national database with deidentified patients, risk to participants is minimal. There is no consent required.

\section{References}

1. Centers for Disease C, Prevention. Consumption of cigarettes and combustible tobacco-United States, 2000-2011. MMWR Morb Mortal Wkly Rep. 2012;61(30):565-9.

2. Moolgavkar SH, Holford TR, Levy DT, Kong CY, Foy M, Clarke $\mathrm{L}$, et al. Impact of reduced tobacco smoking on lung cancer mortality in the United States during 1975-2000. J Natl Cancer Inst. 2012;104(7):541-8. https://doi.org/10.1093/jnci/djs136.

3. Ockene IS, Miller NH. Cigarette smoking, cardiovascular disease, and stroke: a statement for healthcare professionals from the American Heart Association. American Heart Association Task Force on Risk Reduction. Circulation. 1997;96(9):3243-7.

4. National Center for Chronic Disease P, Health Promotion Office on S, Health. Reports of the Surgeon General. Preventing tobacco use among youth and young adults: a report of the Surgeon General. Reports of the Surgeon General. Atlanta: Centers for Disease Control and Prevention (US); 2012.

5. Health UDo, Services H. The health consequences of smoking - 50 years of progress: a report of the Surgeon General, vol. 17. Atlanta, GA: US Department of Health and Human Services, Centers for Disease Control and Prevention, National Center for Chronic Disease Prevention and Health Promotion, Office on Smoking and Health; 2014. p. 55-71.

6. Kelly TL, Gilpin E, Ahnve S, Henning H, Ross J Jr. Smoking status at the time of acute myocardial infarction and subsequent prognosis. Am Heart J. 1985;110(3):535-41.

7. Ali SF, Smith EE, Bhatt DL, Fonarow GC, Schwamm LH. Paradoxical association of smoking with in-hospital mortality among patients admitted with acute ischemic stroke. J Am Heart Assoc. 2013;2(3):e000171.

8. Ovbiagele B, Saver JL. The smoking - thrombolysis paradox and acute ischemic stroke. Neurology. 2005;65(2):293-5.

9. Purcell IF, Newall N, Farrer M. Lower cardiac mortality in smokers following thrombolysis for acute myocardial infarction may be related to more effective fibrinolysis. QJM Mon J Assoc Physicians. 1999;92(6):327-33. https://doi.org/10.1093/qjmed 192.6.327.

10. Angeja BG, Kermgard S, Chen MS, McKay M, Murphy SA, Antman EM, et al. The smoker's paradox: insights from the angiographic substudies of the TIMI trials. J Thromb Thrombolysis. 2002;13(3):133-9.
11. Barua RS, Sy F, Srikanth S, Huang G, Javed U, Buhari C, et al. Acute cigarette smoke exposure reduces clot lysis-association between altered fibrin architecture and the response to t-PA. Thromb Res. 2010;126(5):426-30. https://doi.org/10.1016/j.throm res.2010.07.021.

12. Giordano FJ. Oxygen, oxidative stress, hypoxia, and heart failure. J Clin Investig. 2005;115(3):500-8. https://doi.org/10.1172/JCI24 408.

13. Lacasse Y, LaForge J, Maltais F. Got a match? Home oxygen therapy in current smokers. London: BMJ Publishing Group Ltd; 2006.

14. Chi JH, Knudson MM, Vassar MJ, McCarthy MC, Shapiro MB, Mallet $\mathrm{S}$, et al. Prehospital hypoxia affects outcome in patients with traumatic brain injury: a prospective multicenter study. J Trauma. 2006;61(5):1134-41. https://doi.org/10.1097/01.ta.00001 96644.64653.d8.

15. Spaite DW, Hu C, Bobrow BJ, Chikani V, Barnhart B, Gaither $\mathrm{JB}$, et al. The effect of combined out-of-hospital hypotension and hypoxia on mortality in major traumatic brain injury. Ann Emerg Med. 2017;69(1):62-72. https://doi.org/10.1016/j.annemergme d.2016.08.007.

16. Nahmias J, Doben A, Poola S, Korntner S, Carrens K, Gross R. Implementation of a quality improvement project on smoking cessation reduces smoking in a high risk trauma patient population. World J Emerg Surg. 2016;11(1):15. https://doi.org/10.1186/ s13017-016-0072-7.

17. Ferro TN, Goslar PW, Romanovsky AA, Petersen SR. Smoking in trauma patients: the effects on the incidence of sepsis, respiratory failure, organ failure, and mortality. J Trauma. 2010;69(2):308 12. https://doi.org/10.1097/TA.0b013e3181e1761e.

18. Bell TM, Bayt DR, Zarzaur BL. "Smoker's Paradox" in patients treated for severe injuries: lower risk of mortality after trauma observed in current smokers. Nicotine Tob Res. 2015;17(12):1499-504. https://doi.org/10.1093/ntr/ntv027.

19. Flagel BT, Luchette FA, Reed RL, Esposito TJ, Davis KA, Santaniello JM, et al. Half-a-dozen ribs: the breakpoint for mortality. Surgery. 2005;138(4):717-23. https://doi.org/10.1016/j. surg.2005.07.022 (discussion 23-5).

20. Barnea Y, Kashtan H, Skornick Y, Werbin N. Isolated rib fractures in elderly patients: mortality and morbidity. Can J Surg. 2002;45(1):43-6.

21. May L, Hillermann C, Patil S. Rib fracture management. BJA Educ. 2015;16(1):26-32.

22. Kaijser L, Berglund B. Effect of nicotine on coronary blood-flow in man. Clin Physiol (Oxford, England). 1985;5(6):541-52.

23. Poloujadoff MP, Borron SW, Amathieu R, Favret F, Camara MS, Lapostolle F, et al. Improved survival after resuscitation with norepinephrine in a murine model of uncontrolled hemorrhagic shock. Anesthesiology. 2007;107(4):591-6. https://doi. org/10.1097/01.anes.0000281926.54940.6a.

24. Zhu BQ, Parmley WW. Hemodynamic and vascular effects of active and passive smoking. Am Heart J. 1995;130(6):1270-5.

25. Shi Y, Weingarten TN, Mantilla CB, Hooten WM, Warner DO. Smoking and pain pathophysiology and clinical implications. Anesthesiol J Am Soc Anesthesiol. 2010;113(4):977-92.

26. Council NR. Environmental tobacco smoke: measuring exposures and assessing health effects. Washington, D.C.: National Academies Press; 1986.

27. Sangani RG, Ghio AJ. Lung injury after cigarette smoking is particle related. Int J Chronic Obstr Pulm Dis. 2011;6:191-8. https ://doi.org/10.2147/Copd.S14911.

28. Saha SP, Bhalla DK, Whayne TF Jr, Gairola C. Cigarette smoke and adverse health effects: an overview of research trends and future needs. Int J Angiol. 2007;16(3):77-83.

29. Petrusca DN, Van Demark M, Gu Y, Justice MJ, Rogozea A, Hubbard WC, et al. Smoking exposure induces human lung 
endothelial cell adaptation to apoptotic stress. Am J Respir Cell Mol Biol. 2014;50(3):513-25. https://doi.org/10.1165/rcmb.20130023OC.

30. Barnes MA, Carson MJ, Nair MG. Non-traditional cytokines: how catecholamines and adipokines influence macrophages in immunity, metabolism and the central nervous system. Cytokine. 2015;72(2):210-9. https://doi.org/10.1016/j.cyto.2015.01.008.

31. Grebe KM, Takeda K, Hickman HD, Bailey AL, Embry AC, Bennink JR, et al. Cutting edge: sympathetic nervous system increases proinflammatory cytokines and exacerbates influenza A virus pathogenesis. J Immunol. 2010;184(2):540-4. https://doi. org/10.4049/jimmunol.0903395.

32. Broug-Holub E, Persoons JH, Schornagel K, Mastbergen SC, Kraal G. Effects of stress on alveolar macrophages: a role for the sympathetic nervous system. Am J Respir Cell Mol Biol. 1998;19(5):842-8. https://doi.org/10.1165/ajrcmb.19.5.3103.

33. Calfee CS, Matthay MA, Eisner MD, Benowitz N, Call M, Pittet $\mathrm{JF}$, et al. Active and passive cigarette smoking and acute lung injury after severe blunt trauma. Am J Respir Crit Care Med. 2011;183(12):1660-5. https://doi.org/10.1164/rccm.20101 1-1802OC.

34. Panzer AR, Lynch SV, Langelier C, Christie JD, McCauley K, Nelson $\mathbf{M}$, et al. Lung microbiota is related to smoking status and to development of acute respiratory distress syndrome in critically ill trauma patients. Am J Respir Crit Care Med. 2018;197(5):62131. https://doi.org/10.1164/rccm.201702-0441OC.

35. Resnick S, Inaba K, Okoye O, Nosanov L, Grabo D, Benjamin E, et al. Impact of smoking on trauma patients. Turk J Trauma Emerg Surg. 2014;20(4):248-52. https://doi.org/10.5505/tjtes .2014.21737.

36. Brasel KJ, Guse CE, Layde P, Weigelt JA. Rib fractures: relationship with pneumonia and mortality. Crit Care Med. 2006;34(6):1642-6. https://doi.org/10.1097/01.CCM.0000217926 $.40975 .4 \mathrm{~B}$
37. Lien YC, Chen $\mathrm{CH}$, Lin HC. Risk factors for 24-hour mortality after traumatic rib fractures owing to motor vehicle accidents: a nationwide population-based study. Ann Thorac Surg. 2009;88(4):1124-30. https://doi.org/10.1016/j.athoracsur 2009.06.002.

38. Battle CE, Hutchings H, Evans PA. Risk factors that predict mortality in patients with blunt chest wall trauma: a systematic review and meta-analysis. Injury. 2012;43(1):8-17. https://doi. org/10.1016/j.injury.2011.01.004.

39. Sobrino J, Shafi S. Timing and causes of death after injuries. In: Baylor University Medical Center Proceedings, vol. 26, no. 2. Taylor \& Francis; 2013. p. 120-3.

40. Aziz M, Jacob A, Yang WL, Matsuda A, Wang P. Current trends in inflammatory and immunomodulatory mediators in sepsis. J Leukoc Biol. 2013;93(3):329-42. https://doi.org/10.1189/ jlb.0912437.

41. Kak V. Mediators of systemic inflammatory response syndrome and the role of recombinant activated protein $\mathrm{C}$ in sepsis syndrome. Infect Dis Clin N Am. 2011;25(4):835-50. https://doi. org/10.1016/j.idc.2011.07.009.

42. Wittebole X, Hahm S, Coyle SM, Kumar A, Calvano SE, Lowry SF. Nicotine exposure alters in vivo human responses to endotoxin. Clin Exp Immunol. 2007;147(1):28-34. https://doi.org/10 $.1111 / j .1365-2249.2006 .03248 . x$.

43. Hofer S, Eisenbach C, Lukic IK, Schneider L, Bode K, Brueckmann $\mathrm{M}$, et al. Pharmacologic cholinesterase inhibition improves survival in experimental sepsis. Crit Care Med. 2008;36(2):404-8. https://doi.org/10.1097/01.CCM.0B013E31816208B3.

44. Wang H, Liao H, Ochani M, Justiniani M, Lin X, Yang L, et al. Cholinergic agonists inhibit HMGB1 release and improve survival in experimental sepsis. Nat Med. 2004;10(11):1216-21. https:// doi.org/10.1038/nm1124. 